\title{
Refinement of crystal structure using 'digital' large angle convergent beam electron diffraction
}

\author{
Richard Beanland, Alex Hubert and Rudo Roemer \\ University of Warwick, England, United Kingdom
}

Electron diffraction has long suffered the perception of being unreliable when it comes to structure solution and refinement, coming in a poor third place to more straightforward methods such as X-ray and neutron diffraction. While it has the strong advantages of being applicable to nanoscale volumes and higher sensitivity to light elements, the high scattering cross-section of electrons interacting with atoms also results in multiple scattering. This changes diffracted intensities enormously and cannot be ignored. There have been three main barriers to progress, all of which are now very much reduced due to improvements in technology: i) the ability to automate data collection; ii) uncertainties in crystal orientation; and iii) the relatively difficult, and therefore slow, calculation of scattered intensities taking account of multiple scattering. Computer control, digitised detector technology, and improvements in computation power effectively solve the first and last issues, while collection of sufficient amounts of data solve the second.

Here, we examine 'digital' large angle convergent beam electron diffraction (D-LACBED) and its sensitivity to different experimental and material parameters. D-LACBED data overcome the limitation imposed by the very small Bragg angle for high energy electron diffraction, that limits the angular range of the incident beam when collecting a Kossel-Mollenstedt convergent beam electron diffraction (CBED) pattern. By collecting hundreds, or thousands, of individual CBED patterns, each of which has a small angular range of 0.1 to $1.0 \mathrm{mrad}$, followed by rearranging the data, patterns covering a relatively large angular range of 20-40 mrad can be assembled for each diffracted beam [1]. An example is given in Fig. 1 , which shows the reconstruction of the 200 LACBED pattern at the [001] zone axis of $\mathrm{BaTiO}_{3}$. The outer figure shows an individual CBED pattern with the 000 beam tilted some way from the zone axis; the sequence of CBED patterns collected at the beam tilt is rastered is shown by a series of white outlines. The inset shows the reconstructed 200 LACBED pattern with the same sequence of beam positions. Effectively, each disc in the CBED pattern is a window onto a larger LACBED pattern that usually cannot be seen in its entirety because of the restriction on beam convergence angle. For the data shown in Fig. 1, 961 individual CBED patterns allowed the reconstruction of 121 D-LACBED patterns. The reconstructed D-LACBED data contains a great deal of detail showing dynamical diffraction effects.

The intensities in these patterns are sensitive to six main parameters, two of which are experimental (specimen thickness and accelerating voltage) and four determined by the material: atomic coordinates, occupancy, lattice parameter and temperature (Debye-Waller) factors. In practice a seventh parameter must be included in any refinement procedure - the angular range of the D-LACBED pattern - since this is rarely known with sufficient accuracy. The sensitivity of the data varies significantly for the different parameters, as shown on the left of Fig. 2 for the [2-21] zone axis of corundum $\left(\mathrm{Al}_{2} \mathrm{O}_{3}\right)$.[2] Here, Bloch wave simulations [3] are used to calculate the changes in intensity in D-LACBED patterns that result from a change in these seven different parameters. These simulations are for LACBED patterns of size 296x296 pixels covering an angular range of $55.1 \mathrm{mrad}$ and an accelerating voltage of $200 \mathrm{kV}$. The first 49 LACBED patterns $( \pm 3 g)$ were calculated, but only three are shown here: $-114,012$ and -228 . Blue indicates an increase, and orange a decrease, in intensity produced by a positive change in the relevant 
parameter. Importantly, the complex changes in intensity produced by the different parameters vary significantly in the different LACBED patterns. For example, the effect of a change in occupancy on the 012 LACBED pattern has a similar effect to a change in thickness. However, the same changes produce completely different effects in the 000 or -228 patterns. These distinctive changes in intensity effectively allow each parameter to be determined independently. The bottom row of Fig. 2 gives the change in parameter that produces a change in intensity of one standard deviation of the image $\sigma$ (i.e. the square root of the variance of the pixel values in each LACBED pattern). This allows a quantitative comparison of the effect of the different parameters, even though the changes they produce are different. The typical precision that a match between simulation and experiment reaches is approx. $\sigma / 3$, as shown on the right of Fig. 2. Here a map of fit between experiment and simulation for a range of atomic coordinates of $\mathrm{Al}$ and $\mathrm{O}$ is shown. The best fit is obtained at $\mathrm{zAl}=0.3525(3), \mathrm{xO}_{\mathrm{O}}=0.6932(7)$, which is in good agreement with that determined by X-rays [4], i.e. $\mathrm{zAl}_{\mathrm{Al}}=0.352156(17)$, $\mathrm{xO}=0.69364(7)$, a difference of 0.4 and 0.2 picometres respectively.

Applying the same criterion to the other parameters, we may expect specimen thickness to be determined with a precision of $0.6 \mathrm{~nm}$, occupancy $0.6 \%$, unit cell dimensions to $1.2 \mathrm{pm}$, accelerating voltage $2.5 \mathrm{kV}$ and temperature (B-factors) to $0.1 \AA^{2}$.

In summary, D-LACBED data exhibits a high sensitivity to several useful and interesting parameters that should allow them to be determined with high precision.

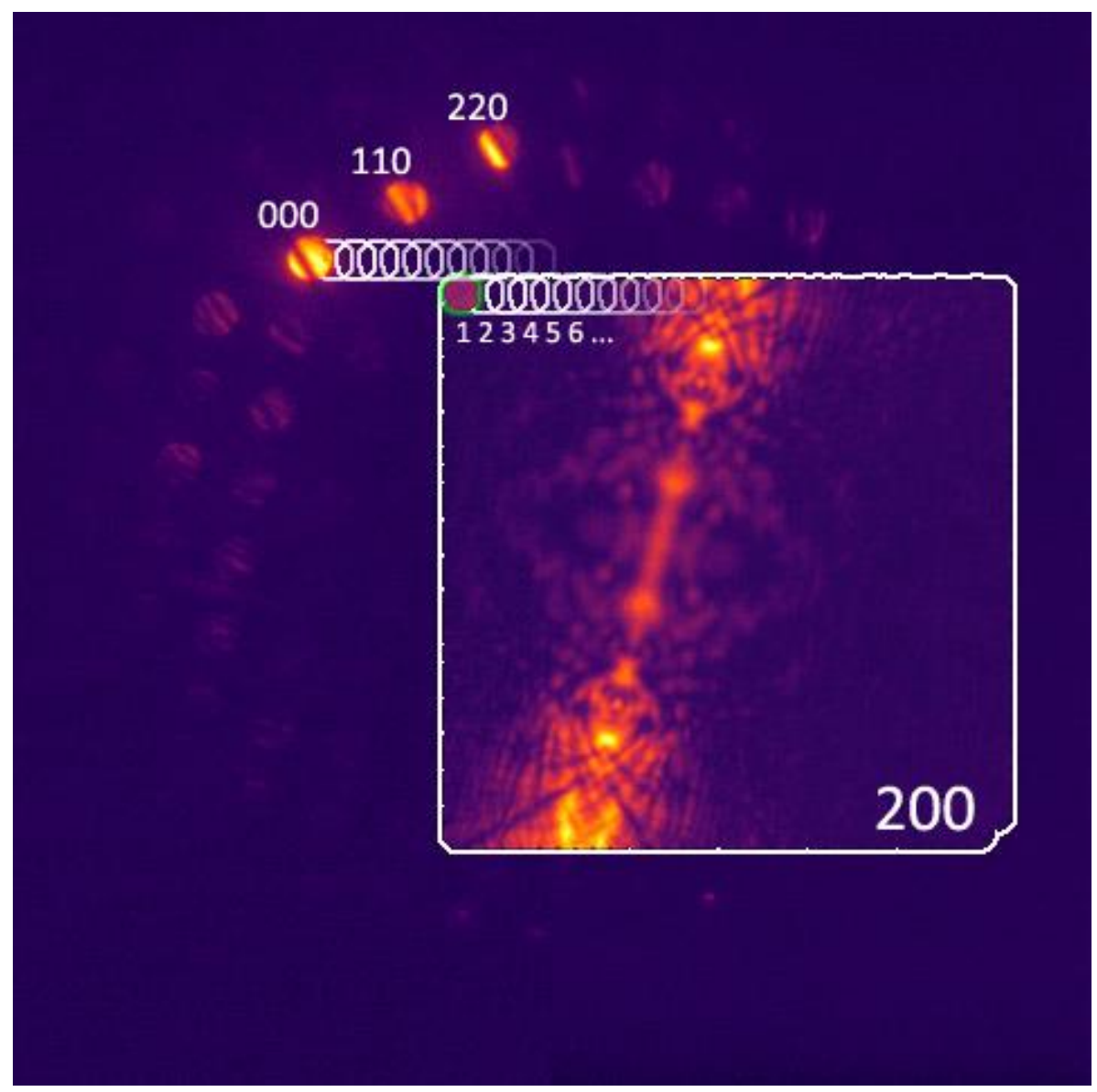

Figure 1. Reconstruction of the 200 D-LACBED pattern at the [001] zone axis of BaTiO3. 

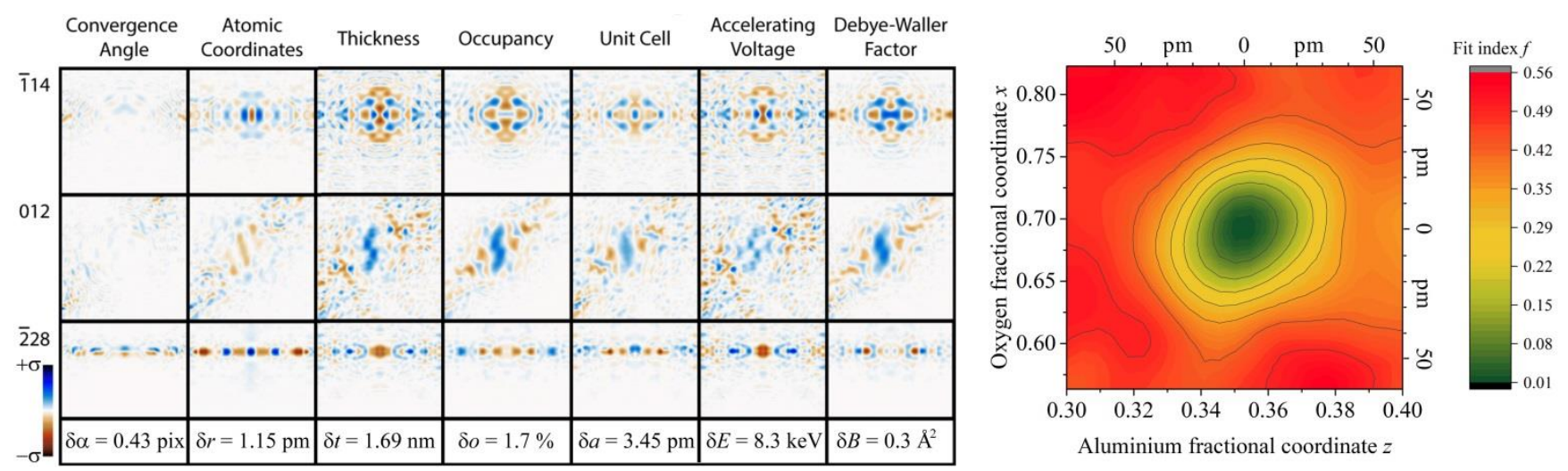

Figure 2. Left: Changes in intensity in three LACBED patterns at the Al2O3 [2-21] zone axis, calculated using Bloch wave simulations.[3] Right: map of fit parameter for changes in Al and O coordinates.

\section{References}

[1] Beanland, R., Thomas, P.J., Woodward, D.I., Thomas, P.A. and Roemer, R.A., 2013. Digital electron diffraction-seeing the whole picture. Acta Crystallographica Section A: Foundations of Crystallography, 69(4), pp.427-434.

[2] Hubert, A.J.M., Römer, R. and Beanland, R., 2019. Structure refinement from 'digital'large angle convergent beam electron diffraction patterns. Ultramicroscopy, 198, pp.1-9.

[3] Beanland, R., Evans, K., Roemer, Rudolf A., and Hubert, A. J. M., FELIX Bloch wave simulation: Source code https://github.com/RudoRoemer/Felix

[4] Kondo, S., Tateishi, K. and Ishizawa, N., 2008. Structural evolution of corundum at high temperatures. Japanese Journal of Applied Physics, 47(1S), p.616. 